\title{
Study of Relationship between Health, Integrity and Depression in the Elderly Population: A Salutogenic Approach
}

\author{
Romil Singh ${ }^{1}$, Richa Sharma ${ }^{2}$ \\ ${ }^{1}$ Junior Practitioner, Metropolitan Hospital, Jaipur, Rajasthan, India, ${ }^{2}$ Director, Regional Institute of Health, Medicine \& Research, Jaipur,Rajasthan, India.
}

\section{Abstract}

Background: The salutogenesis is very relevant concept in today's era when we are shifting focus from tertiary to primordial prevention where it can show us a way to remain healthy and act as a concept of pre-primordial prevention. These apparent links between the sense of coherence which defines the integrity of an individual, depression which is a consequence of changed demands of life and its implications on the health of an individual will provide a conceptual framework to this study. Subjects and Methods: This is a cross sectional community based study done on above 60 years of age from an urban area which is a field practice area of the institution taken as study group A and elderly residing in the old age homes of the city taken as study group B. They were explained about the details of filling the questionnaires in advance and their identity were kept confidential and the participation was voluntary.Orientation to life questionnaire i.e. OLQ-13 version was used to estimate sense of coherence scores of the individual.GDS or geriatric depression scale is an appropriate questionnaire used for measuring the depressional status of an elderly person in their respective life stressful conditions. Results: Our study showed that the $61.7 \%$ oftotalpopulationofstudygroupAweremalesand $38.33 \%$ werefemales. $15 \%$ ofthetotalelderlyofstudygroupAhavehighsoc, $84.17 \%$ havemediu msocand $1 \%$ have lowsoc. $13.33 \%$ of the elderly in study group B have high SOC whereas $87 \%$ have medium SOC.Conclusion: We concluded that SOC and BMI are directly proportional to each other; GDS and education were in direct correlation to each other; SOC was found to be directly proportional to the educational status of theperson.

Keywords: Depression, Elderly people, SOC, GDS.

Corresponding Author: Dr. Richa Sharma, Director, Regional Institute of Health, Medicine \& Research, Jaipur, Rajasthan, India.

Received: January 2020

Accepted: January 2020

\section{Introduction}

Elderly persons experience a complex array of physical (poor sleep, pain, low energy, poor concentration, poor appetite), psychological (negative thoughts, low self esteem, sadness, hopelessness) and social changes (social withdrawal, decrease in activity, decreased productivity, lack of initiation. Yet despite high prevalence, depression remains under diagnosed ${ }^{[1]}$ Salutogenesis means genesis of health as opposed to pathogenesis. It was introduced by Dr. Aaron Antonovsky, an American-Israeli sociologist. The concept includes ${ }^{2}$ firstly, the orientation towards problem solving and secondly the capacity to use the resources available. The concept consists of 2 major pillars supporting a healthy life i.e. 1 . Sense of coherence and 2. Generalized resistance resources. ${ }^{[2]}$

Whether a stress factor will be pathogenic, neutral or salutary depends on the generalized resistance resources. The GRR's are of both genetic and constitutional and psychosocial characters such as material, knowledge /intelligence,ego identity, coping strategy (rational, flexible, far sighted), social support, commitment (continuance, cohesion,control),cultural stability and a preventive health orientation. ${ }^{[2,3]}$ GRR's have 2 functions, firstly, they have a continuous effect on life enabling us to make coherent and meaningful decisions in life which in turn form the SOC and secondly, they function as a potential which can be activated when necessary for managing stress and tension. ${ }^{[4]}$

The salutogenesis is very relevant concept in today's era when we are shifting focus from tertiary to primordial prevention where it can show us a way to remain healthy and act as a concept of pre-primordial prevention.

United Nations has ranked India at 134 out of 187 countries in terms of quality of life index/human development index. Within the field of healthcare, quality of life is often regarded in terms of how it is negatively affected, on an individual level. This study aims to change the same i.e. to empower the positive conscience of a person over his negative conscience.

These apparent links between the sense of coherence which defines the integrity of an individual, depression which is a consequence of changed demands of life and its implications on the health of an individual will provide a conceptual framework to this study.

\section{Subjects and Methods}

This is a cross sectional community based study done on above 60 years of age from an urban area which is a field practice area of the institution taken as study group A and 
elderly residing in the old age homes of the city taken as study group B. They were explained about the details of filling the questionnaires in advance and their identity were kept confidential and the participation was voluntary. It was specified that data will be used only for research purposes.

\section{Study Setting}

The elderly people residing in the field study area and in the oldage homes were interviewed and the first part of the performa i.e. "theperforma questioning the personal and general information" was filled by theinterviewer. The remaining two sections i.e. "the orientation to lifequestionnaire and the geriatric depression scale" respectively were filled up by the elderly persons, assistance was provided to the needy ones in filling up thequestionnaire. The scoring of both the questionnaires was done according tothe standard procedures.

\section{Number of Subjects Included}

Atotal of 240 elderly persons were included i.e.120 from old age home residents and 120 from the elderly living in the urbanarea.

\section{Inclusion and Exclusion Criteria}

Persons of age of 60 or above it living in oldage homes or residents of the urban area were included in this study. Theelderly people with any mental problems, deaf and dumb, NRI's , having anyprevious criminal records , suffering from any communicable disease whichmay have affected the results in a negative way were excluded from the study.

\section{Methods}

Orientation to life questionnaire i.e. OLQ-13 version was used to estimatesense of coherence scores of the individual. ${ }^{5}$ In total the SOC questionnaireshas been used in 33 languages in 32 countries. $^{[3]}$ It consists of four items ofmanageability which explains the extent to which the person perceives thatresources at their disposal are adequate to meet the demands posed by stimulithat bombards them, this is the instrumental or behavioral component of SOC. ${ }^{[3]}$ It consists of 5 items of comprehensibility which refers to the personal stimulithat confront us deriving from the external and internal environment. Theperson scoring high on the sense of comprehensibility expects that stimuli theyencounter in the future will be predictable, ordered, and explicit, this is thecognitive component of SOC. ${ }^{[3]}$

4 items for meaningfulness demonstrate the extent to which the person feelsthat life makes sense emotionally, that problems and demands are worthinvesting energy in, are worthy of commitment and engagement, seen aschallenges rather than burdens this is the motivational component of thequestionnaire. ${ }^{[3]}$

The term quality of life is used to evaluate the general wellbeing of individualsand societies including the fields of international development, healthcare, andpolitics. Quality of life should not be confused with the concept of standard ofliving, which is based primarily on income. Instead, standard indicators of thequality of life include not only wealth and employment, but also the builtenvironment, physical and mental health, education, recreation and leisuretime, and social belonging. ${ }^{[2]}$ QOL reflects the difference, the gap, betweenthe hopes and expectations of a person and their present experience. Humanadaptation is such that life expectations are usually adjusted so as to lie withinthe realm of what the individual perceives to be possible. This enables peoplewho have difficult life circumstances to maintain a reasonable QOL.

The necessary language modification in OLQ was done in order to adapt itlocally. All the OLQ items were fitted to a seven point LIKERT scale. Each optionwas explained to the people to make it understandable to them.Each questionhad 7 options ranging from 1 to seven, the first and seventh option being thetwo extremes of a particular question.The subject was told to select any oneoption according to his/her orientation towards life. The scores were thencumulated. Possible scores ranging from 13 to 91 with greater scores indicatinggreater sense of coherence were found, with a mean score of 58 .

\section{Geriatric Depression Scale (GDS)}

GDS or geriatric depression scale is an appropriate questionnaire used formeasuring the depressional status of an elderly person in their respective lifestressful conditions. ${ }^{6}$ The GDS questions are answered in a simple "yes" or"no". This simplicity enables the scale to be used with ill or moderatelycognitively impaired individuals. The geriatric depression scale short form(GDSSF) is a 15-item self rating scale commonly used as a routine part ofcomprehensive geriatric assessment. ${ }^{[7]}$ One point is assigned to each answerand the cumulative score is rated on a scoring grid. The grid sets a range of 0-4as "normal", 5-9 as "mildly depressed", and 10-15 as "severely depressed". Unlike other depression scales for adult population, GDSSF does not includesomatic symptoms because many of these symptoms (for example, sleep disturbance, weight loss, and pessimism about the future) may be related tothe normal process of ageing or to an underlying physical illness. ${ }^{8}$ Manymedical problems common to older people may be related to, or intensified by, a depressive disorder. The GDS was found to have $92 \%$ sensitivity and $89 \%$ specificity when evaluated against diagnostic criteria. The validity andreliability of the tool have been supported through both clinical practice andresearch based on analysis of Yesavageet al. ${ }^{[6]}$

The GDSSF is derived from the 30-item GDS long form (GDSLF), which has highinternal consistency and performance that is comparable to the Hamiltondepression scale. ${ }^{[9]}$ Correlations between the GDSSF and the GDSLF rangefrom 0.66 to $0.89 .{ }^{[7-9]}$ The GDSSF is currently screening tool for most patientsadmitted to the NARG program. ${ }^{[1]}$

\section{Results}

Our study showed that the 61.7\%oftotalpopulationofstudygroupAweremalesand38.33 
\%werefemales.52.5\%ofthe

totalelderlyofstudygroupAbelongedto60-

65agegroupcategory.65\%oftotalpopulation

ofstudygroupBweremalesand $35 \%$ were females [Table 1].

The present study showed that

$15 \%$ ofthetotalelderlyofstudygroupAhavehighsoc, $84.17 \%$ ha

vemediumsocand $1 \%$ have lowsoc (Table 2).13.33\% of the elderly in study group B have high SOC whereas $87 \%$ have medium SOC (table 3).In table $4 \&$ 5showed that $56 \%$ oftheelderlyofstudygroupAhavingmediumSOCarenotde pressedwhereas $9.9 \%$ having

mediumSOCareslightlydepressedand34\%ofthesamearehighl ydepressed.

66\%oftheelderlyofstudygroupBhavingmediumSOCaremode ratelydepressedand $17 \%$ arehighlydepressed.

Our study showed that $1.48 \%$ oftheelderlyhavingmediumSOCwereofnormalweighta ndhealthyand52\%oftheelderly

ofmediumSOCcombined(underweight+overweight+obese)

werenothealthy

[Table

$6]$.

$61 \%$ oftheelderlyhavingmediumSOCwereofnormalweightan dhealthyand $39 \%$ oftheelderly

ofmediumSOCcombined(underweight+overweight+obese) werenot healthy [Table 7].

Table 1: Age and Sex

\begin{tabular}{|l|l|l|l|l|l|l|l|l|l|l|l|l|}
\hline \multicolumn{10}{|c|}{ Study Group A } & \multicolumn{10}{|c|}{$\begin{array}{c}\text { Atudy Group } \\
\text { B }\end{array}$} & \multicolumn{7}{c|}{ Total } \\
\hline AGE & M & F & T & $\%$ & M & F & T & $\%$ & M & F & T & $\%$ \\
\hline $60-65$ & 39 & 24 & 63 & 52.5 & 13 & 6 & 19 & 15.83 & 52 & 30 & 82 & 34.16 \\
\hline $66-70$ & 16 & 15 & 31 & 25.83 & 14 & 11 & 25 & 20.83 & 30 & 26 & 76 & 31.66 \\
\hline more than70 & 19 & 7 & 26 & 21.66 & 61 & 25 & 76 & 63.33 & 80 & 32 & 11246.66 \\
\hline Total & 74 & 46 & 120 & & 78 & 42 & 120 & & 152 & 88 & 240 & \\
\hline$\%$ & 61.66 & 38.33 & & & 65 & 35 & & & 63.33 & 36.66 & & \\
\hline
\end{tabular}

Table 2: SOC and Education in study group A

\begin{tabular}{|c|c|c|c|c|c|c|c|c|c|c|c|c|}
\hline & \multicolumn{12}{|c|}{ Soc } \\
\hline & \multicolumn{12}{|c|}{ Study Group A } \\
\hline & \multicolumn{4}{|c|}{ High } & \multicolumn{4}{|c|}{ Medium } & \multicolumn{2}{|c|}{ Low } & & \\
\hline Education & M & $F$ & $T$ & $\%$ & $\mathrm{M}$ & $\mathrm{F}$ & $T$ & $\%$ & $\mathrm{M}$ & $F$ & $\mathrm{~T}$ & $\%$ \\
\hline Illitrate & & & & & 6 & 26 & 32 & 31.68 & 1 & 0 & 1 & 100 \\
\hline Primary & 1 & 1 & 2 & 11.11 & 5 & 1 & 6 & 5.94 & 0 & 0 & 0 & \\
\hline Secondary & 1 & 0 & 1 & 5.55 & 6 & 5 & 11 & 10.89 & 0 & 0 & 0 & \\
\hline Hr.Sec & 2 & 0 & 2 & 11.11 & 5 & 3 & 8 & 7.92 & 0 & 0 & 0 & \\
\hline Graduate & 2 & 0 & 2 & 11.11 & 4 & 9 & 13 & 14.41 & 0 & 0 & 0 & \\
\hline Post.Gr. & 11 & 1 & 12 & 61.11 & 30 & 1 & 31 & 30.69 & 0 & 0 & 0 & \\
\hline Total No. & 17 & 1 & 18 & 15 & 56 & 45 & 101 & & 1 & 0 & 1 & 100 \\
\hline$\%$ & 94 & 5.6 & & & & 45 & & & & & & \\
\hline
\end{tabular}

\section{Table 3: SOC and Education in study group B}

Table 3: SOC and Education in study group B
\begin{tabular}{|l|l|l|l|l|l|l|l|l|l|l|l|l|}
\hline & \multicolumn{10}{c|}{ SOC } \\
\hline & \multicolumn{10}{|c|}{ High } & \multicolumn{3}{|c|}{ Medium } & \multicolumn{3}{c|}{ Low } \\
\hline & \multicolumn{9}{|c|}{ Higdy Group B } \\
\hline Education & M & F & T & $\%$ & M & F & T & $\%$ & M & F & T & $\%$ \\
\hline Illitrate & 0 & 2 & 2 & 12.5 & 6 & 2 & 8 & 7.54 & 0 & 0 & 0 & 0 \\
\hline Primary & 0 & 0 & 0 & 0 & 4 & 2 & 6 & 5.66 & 0 & 0 & 0 & 0 \\
\hline Secondary & 0 & 0 & 0 & 0 & 8 & 6 & 14 & 13.2 & 0 & 0 & 0 & 0 \\
\hline Hr.Sec & 0 & 0 & 0 & 0 & 4 & 4 & 8 & 7.54 & 0 & 0 & 0 & 0 \\
\hline
\end{tabular}

\begin{tabular}{|c|c|c|c|c|c|c|c|c|c|c|c|c|c|c|}
\hline Graduate & & 6 & 0 & 6 & 37.5 & 32 & 4 & 36 & 33.96 & 0 & To & 0 & 0 & \\
\hline Post.Gr. & & 6 & 2 & 8 & 50 & 12 & 22 & 34 & 32.07 & 0 & 10 & 10 & 0 & \\
\hline Total No & & 12 & 4 & 16 & & 66 & 40 & 106 & & 0 & 0 & c & & \\
\hline$\%$ & & 75 & 25 & & & 62 & 38 & & & 0 & 0 & 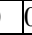 & & \\
\hline \multicolumn{15}{|c|}{ Table 4: SOC and GDS } \\
\hline & \multicolumn{14}{|c|}{ SOC } \\
\hline & \multicolumn{14}{|c|}{ Study Group A } \\
\hline & \multicolumn{5}{|c|}{ High } & \multicolumn{4}{|c|}{\begin{tabular}{|l|} 
Medium \\
\end{tabular}} & \multicolumn{5}{|c|}{ Low } \\
\hline GDS & $M$ & $\mathrm{~F}$ & $T$ & & $\%$ & $M$ & $\mathrm{~F}$ & $T$ & $\%$ & M & $\mathrm{F}$ & $T$ & $\%$ & \\
\hline A & 15 & 1 & 16 & & 88.88 & 41 & 16 & 57 & 56 & 1 & 0 & 1 & - & \\
\hline $\mathrm{B}$ & 2 & 0 & 2 & & 11.2 & 5 & 5 & 10 & 9.9 & 0 & 0 & 0 & - & \\
\hline C & 0 & 0 & 0 & & 0 & 10 & 24 & 34 & 34 & 0 & 0 & 0 & - & \\
\hline TOTAL & & 1 & 18 & & & 56 & 45 & 101 & & 1 & 0 & 1 & - & \\
\hline$\%$ & 89 & 11 & & & & 55.4 & 46 & & & & & & & \\
\hline
\end{tabular}

Table 5: SOC and GDS
\begin{tabular}{|l|l|l|l|l|l|l|l|l|l|l|l|l|}
\hline \multicolumn{10}{c|}{ HOgh } & \multicolumn{3}{c|}{ Medium } \\
\hline & \multicolumn{10}{c|}{ Study Group B } \\
\hline & \multicolumn{10}{|c|}{ High } & \multicolumn{3}{c|}{ Low } \\
\hline GDS & M & F & T & $\%$ & F & T & $\%$ & M & F & T & $\%$ \\
\hline A & 2 & 2 & 4 & 25 & 10 & 8 & 18 & 17 & 0 & 0 & 0 & 0 \\
\hline B & 10 & & 10 & 62.5 & 48 & 22 & 70 & 66 & 0 & 0 & 0 & 0 \\
\hline C & & 2 & 2 & 12.5 & 8 & 10 & 18 & 17 & 0 & 0 & 0 & 0 \\
\hline TOTAL & 12 & 4 & 16 & & 66 & 40 & 106 & & 0 & 0 & 0 & \\
\hline$\%$ & 75 & 25 & & & 62.3 & 38 & & & & & & \\
\hline
\end{tabular}

\begin{tabular}{|c|c|c|c|c|c|c|c|c|c|c|c|c|}
\hline \multicolumn{13}{|c|}{ Table 6: SOC and BMI } \\
\hline & \multicolumn{12}{|c|}{ SOC } \\
\hline & \multicolumn{12}{|c|}{ Study Group A } \\
\hline & \multicolumn{4}{|c|}{ High } & \multicolumn{4}{|c|}{ Medium } & \multicolumn{4}{|c|}{ Low } \\
\hline BMI & $\mathrm{M}$ & $F$ & $\mathrm{~T}$ & $\%$ & M & $\mathrm{F}$ & $\mathrm{T}$ & $\%$ & $\mathrm{M}$ & $\mathrm{F}$ & $\mathrm{T}$ & $\%$ \\
\hline less than 18.5 & & 0 & 1 & 5.55 & 1 & 4 & 5 & 5 & 0 & 0 & 0 & 0 \\
\hline $18.5-25$ & 14 & 0 & 14 & 77.8 & 25 & 23 & 48 & 48 & 0 & 0 & 0 & 0 \\
\hline $25-30$ & 2 & 1 & 3 & 16.7 & 29 & 16 & 45 & 45 & 1 & 0 & 1 & 100 \\
\hline more than 30 & 0 & 0 & 0 & 0 & 1 & 3 & 4 & 4 & 0 & 0 & 0 & 0 \\
\hline Total & 17 & 1 & 18 & & 56 & 45 & 101 & & 1 & 0 & 1 & \\
\hline$\%$ & 94 & 6 & & & 55.4 & 45 & & & 100 & 0 & & \\
\hline
\end{tabular}

Table 7: SOC and BMI
\begin{tabular}{|l|l|l|l|l|l|l|l|l|l|l|l|l|}
\hline \multicolumn{10}{c|}{ High } & \multicolumn{7}{c|}{ Medium } \\
\hline \multicolumn{10}{|c|}{ Study Group B } \\
\hline & \multicolumn{10}{|c|}{ Low } \\
\hline BMI & M & F & T & $\%$ & M & F & T & $\%$ & M & F & T & $\%$ \\
\hline $\begin{array}{l}\text { less } \\
\text { than } 18.5\end{array}$ & 0 & 0 & 0 & 0 & 11 & 5 & 16 & 15 & 0 & 0 & 0 & 0 \\
\hline $18.5-25$ & 11 & 3 & 14 & 87.5 & 38 & 25 & 63 & 61 & 0 & 0 & 0 & 0 \\
\hline $25-30$ & 1 & 1 & 2 & 12.5 & 17 & 8 & 25 & 24 & 0 & 0 & 0 & 0 \\
\hline more than 30 & 0 & 0 & 0 & 0 & 0 & 0 & 0 & 0 & 0 & 0 & 0 & 0 \\
\hline Total & 12 & 4 & 16 & & 66 & 38 & 104 & & 0 & 0 & 0 & 0 \\
\hline$\%$ & 75 & 25 & & & 63.5 & 37 & & & & & & \\
\hline
\end{tabular}

\section{Discussion}

The health and well-being of older adults is affected by the level of social activity and the mood states. Researchers have reported the negative effects of loneliness on health in old age (Henkin Yet al., 1996) ${ }^{[10]}$ Loneliness, coupled with other physical and mental problems, gives rise to feelings of depression in the elderly persons. Gender differences have been reported in the prevalence of health problems in 
elderly persons (Arber \&Ginn, 1993). ${ }^{[11]}$ Results in table 1 shows that there is no gender biased done in the study as the number of male and female subjects in the study groups A \& B respectively are nearly equal. As well as in the age categories i.e. $34.16 \%$ belong in 60 65 age group;31.66\% in 66-70 age group and $46.66 \%$ belong to greater than 70 age group. Therefore there are no gender and age differences in the study.

There is direct correlation between the SOC and GDS as $34 \%$ of medium SOC of study group a are severely depressed and $66 \%$ of the elderly of medium SOC of study group B are moderately depressed. So the depression status of the elderly living in old age is more than those who live in the urban area. In socio economic classification, out of the total people living in the old age home $31.66 \%$ are from the middle class family as compared to $12.5 \%$ living in the urban area. This may be possibly because of the low per capita income in the middle class family the elderly are not properly supported by their kith and kin and are forced to move out to old agehome.

$65 \%$ of the illiterates in the study group A are severely depressed whereas only $5.4 \%$ come under this category as shown in our study. The probable explanation to this is that more the education, the more is the selfdependence. As more education will provide them more earnings and they can support themselves and their spouses in a better way as done by a noneducatedperson. $83 \%$ of the post graduates have excellent self rated health whereas only $55 \%$ of the illiterates have poor self rated health; this may be because of the fact that the post graduates due to their education status have better living conditions as compared to the illiterates. As well as the more educated are more aware about the different health programs and camps conducted by the government and the private sector as compared to that of theilliterates.

It was earlier concluded that $59.33 \%$ of male elderly and $40.66 \%$ of female elderly were within normal range of BMI, $58.8 \%$ of male elderly and $64.63 \%$ of female elderly were in overweight range and $57.43 \%$ of male and $65.5 \%$ of female were placed in obese

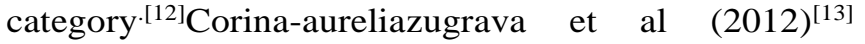
which Results similar to the review of literature were found in this study as $51.21 \%$ of males and $49.39 \%$ of females are in normal BMI range. However there is some difference in the results of BMI in medium SOC for overweight candidates as only $39.34 \%$ of male elderly and $28.91 \%$ of the females are overweight. This may be due to the fact that due to medium and high SOC there is more response to the surroundings and the comprehensibility, meaningfulness factor increases which helps them to stayfit.

\section{Conclusion}

We concluded that SOC and BMI are directly proportional to each other; GDS and education were in direct correlation to each other; SOC was found to be directly proportional to the educational status of theperson.

\section{References}

1. NIH consensus development panel. Diagnosis and treatment of depression in late life. JAMA. 1992; 268(8):1018-29.

2. Antonovsky A. Health, stress, coping.San Francisco. Josseybass1979.

3. Eriksson, Bengt Lindstrom. Validity of Antonovsky's sense of coherence scale: a systemic review. J Epedemiol Community Health. 2005;59(6):460:66.

4. Jurgen Bengal, Regine Stritmatter and Hildegard Willmann.The current state of discussion and the relevance of Antonovskysalutogenic model of health; federal centre for health education. 1999; Volume 4.

5. Dudek B, MakowskaZ. Psychiatric characterstic of the orientation to life questionnaire for measuring sense of coherence. Polish Psychological Bulliten. 1993;24:304-18.

6. Yesavage ,J,A,Brink, T.L. , Lum., O. Huang. Development and validation of a GDS: A Preliminary report journal of psychiatric research.1982-83; 17(1): 37- 49

7. Sheikh JI, Yesavage JA. Geriatric Depression Scale (GDS): recent evidence and development of a shorter version. ClinGerontol 1986;5:65-172.

8. McDowell I, Newell C. Measuring health: a guide to rating scales and questionnaires. 2nd ed. New York (NY): Oxford University Press; 1996.

9. Ngo-Metzger Q, Sorkin DH, Mangione CM, Gandek B, Hays RD. Evaluating the SF-36 Health Survey (Version 2) in Older Vietnamese Americans J Aging Health. 2008 Jun; 20(4): 420-36.

10. Henkin Y, Sperber AD. Aaron Antonovsky: Editor and Idealist. Israel Journal of Medical Sciences. 1996; 32(3-4):163-5.

11. Arber S, Ginn J. Gender and inequalities in health in later life.SocSci Med. 1993 Jan;36(1):33-46.

12. BMI Classification. Global database on body mass index. WHO2006. Retrivedjuly 27,2012.

13. Corelia-aureliaZugrava- Sense of coherence and its connections with BMI and weight-related beliefs andattitudes. International Journal of Collaborative Research on Internal Medicine\& Public Health. 2012; 4(6):1131-40.

Copyright: (C) the author(s), 2020. It is an open-access article distributed under the terms of the Creative Commons Attribution License (CC BY 4.0), which permits authors to retain ownership of the copyright for their content, and allow anyone to download, reuse, reprint, modify, distribute and/or copy the content as long as the original authors and source are cited.

How to cite this article: Singh R, Sharma R.Study of Relationship between Health, Integrity and Depression in the Elderly Population: A Salutogenic Approach. Asian J. Med. Res. 2020;9(1):CM07-CM10.

DOI: dx.doi.org/10.47009/ajmr.2020.9.1.CM3

Source of Support: Nil, Conflict of Interest: None declared. 